\title{
Splitting of the giant monopole and quadrupole resonances in ${ }^{154} \mathrm{Sm}$
}

\author{
D. H. Youngblood, Y.-W. Lui, and H. L. Clark \\ Cyclotron Institute, Texas A\&M University, College Station, Texas 77843
}

(Received 17 June 1999; published 15 November 1999)

\begin{abstract}
Strength functions for the isoscalar giant monopole and quadrupole resonances in ${ }^{154} \mathrm{Sm}$ have been measured with inelastic scattering of $240 \mathrm{MeV} \alpha$ particles at small angles. The $E 0$ strength distribution containing $\left(104_{-20}^{+15}\right) \%$ of the energy-weighted sum rule (EWSR) is consistent with two peaks at $E_{x}=12.1 \pm 0.4 \mathrm{MeV}$ and $15.5 \pm 0.3 \mathrm{MeV}$ containing (36 \pm 10$) \%$ and $(68 \pm 9) \%$ of the EWSR and the E2 strength distribution containing $\left(103_{-20}^{+18}\right) \%$ of the EWSR is consistent with three peaks at $E_{x}=11.3 \pm 0.2 \mathrm{MeV}, 14.5 \pm 0.5 \mathrm{MeV}$, and $17.5 \pm 0.5 \mathrm{MeV}$ containing $(44 \pm 7) \%,(44 \pm 8) \%$, and $(15 \pm 8) \%$, respectively, of the EWSR.

[S0556-2813(99)04812-8]

PACS number(s): 25.55.Ci, 24.30.Cz, 27.70.+q
\end{abstract}

It was shown a number of years ago that the isoscalar giant quadrupole (GQR) [1,2] and giant monopole resonances (GMR) $[3,4]$ broaden and/or split in deformed nuclei. The GQR splits because the oscillation occurs with orientations along the different axes, while the GMR splits because of interference with the GQR. Kishimoto et al. [1] described the broadening of the GQR in ${ }^{154} \mathrm{Sm}$ as a splitting into different (unresolved) $K$ components, (0, 1, and 2) and, using the schematic model of Mottelsen and applying a rigorous self-consistency, got agreement with the data with a modified quadrupole-quadrupole interaction. A related calculation was carried out by Garg et al. [3] for the GMR assuming it split into $K=0$ and 2 components. Several authors had reported calculations of GQR splitting $[5,6]$ and a quasiparticle random-phase approximation calculation by Zawischa, Speth, and Pal [7] had shown splitting of both the GQR and GMR. More complete theoretical descriptions [8,9] have changed the details of the splitting somewhat, but the existing data were not adequate to test the models. Miura and Torizuka [2], using electron scattering, observed four peaks in the giant resonance region in ${ }^{181} \mathrm{Ta}$ whose properties were consistent with either $2^{+}$or $0^{+}$. They assumed that the lower three peaks were $2^{+}(K=0,1,2$ components $)$ and the highest was the GMR. Morsch et al. [4], using inelastic $\alpha$ scattering, observed three peaks in the giant resonance region in both ${ }^{232} \mathrm{Th}$ and ${ }^{238} \mathrm{U}$ that had angular distributions consistent with $E 0$ or E2. They assumed that the lowest and highest peaks were the two components of the GMR, while the middle peak was the GQR. Neither of these works could distinguish $E 0$ from $E 2$. de Leo et al. [10] obtained an E0 strength function for ${ }^{238} \mathrm{U}$ identifying fission fragments following excitation with small-angle inelastic $\alpha$ scattering and reported a splitting of the $E 0$ strength in rough agreement with the calculations of Abgrall et al. [8]. There have been no results reported where the actual splitting was observed and where the $E 0$ and $E 2$ components were both separately identified.

We have investigated the giant resonance region in ${ }^{154} \mathrm{Sm}$ (where $\beta \sim 0.3$ ) using inelastic scattering of $240 \mathrm{MeV} \alpha$ particles where excellent peak to continuum ratios are obtained [11-13] and where the competing pickup-breakup reactions are well above the region where GQR and GMR strength is expected. In lighter nuclei, we have been able to obtain $E 0$ and E2 strength functions [12,13] and in ${ }^{154} \mathrm{Sm}$ this would allow detailed comparison to the models for both $E 0$ and $E 2$ splitting.

The experimental technique has been described thoroughly in Refs. [11] and [12] and is summarized briefly below. A beam of $240 \mathrm{MeV} \alpha$ particles from the Texas A\&M K500 superconducting cyclotron bombarded a selfsupporting foil $3.8 \mathrm{mg} / \mathrm{cm}^{2}$ thick enriched to $99.5 \%$ in ${ }^{154} \mathrm{Sm}$ located in the target chamber of the multipole-dipolemultipole spectrometer. The beam was delivered to the spectrometer through a beam analysis system having two bends of $88^{\circ}$ and $87^{\circ}$. The beam was limited by slits after the first bend, and the second bend was used for clean up, with slits located so as not to intercept the primary beam. The horizontal acceptance of the spectrometer was $4^{\circ}$ and ray tracing was used to reconstruct the scattering angle. The vertical acceptance was set at $\pm 2^{\circ}$. When the spectrometer central angle $\left(\theta_{\text {spec }}\right)$ was set to $0^{\circ}$, the beam passed beside the detector and was stopped in a carbon block behind the detector. At $\theta_{\text {spec }}=0^{\circ}$, runs with an empty target frame showed $\alpha$ particles uniformly distributed in position at a rate about $1 / 2000$ of that with a target in place.

The focal plane detector covered approximately $55 \mathrm{MeV}$ of excitation from $7 \mathrm{MeV}<E_{x}<62 \mathrm{MeV}$ and measured position and angle in the scattering plane. The out-of-plane scattering angle was not measured. Position resolution of approximately $0.9 \mathrm{~mm}$ and scattering angle resolution of about $0.09^{\circ}$ were obtained.

Each data set was divided into ten angle bins, each corresponding to $\sim 0.4^{\circ}$ using the angle obtained from ray tracing. $\phi$ is not measured by the detector, so the average angle for each bin was obtained by integrating over the height of the solid angle defining slit and the width of the angle bin. Cross sections were obtained from the charge collected, target thickness, dead time, and known solid angle. The cumulative uncertainties in target thickness, solid angle, etc. result in about a $\pm 10 \%$ uncertainty in absolute cross sections. Data were taken with ${ }^{12} \mathrm{C},{ }^{24} \mathrm{Mg}$, and ${ }^{28} \mathrm{Si}$ targets at $\theta_{\text {spec }}=3.5^{\circ}$ at the actual field settings used in the experiments to obtain an energy calibration.

Spectra obtained for two angles are shown in Fig. 1. The giant resonance peak can be seen extending up past $E_{x}$ 

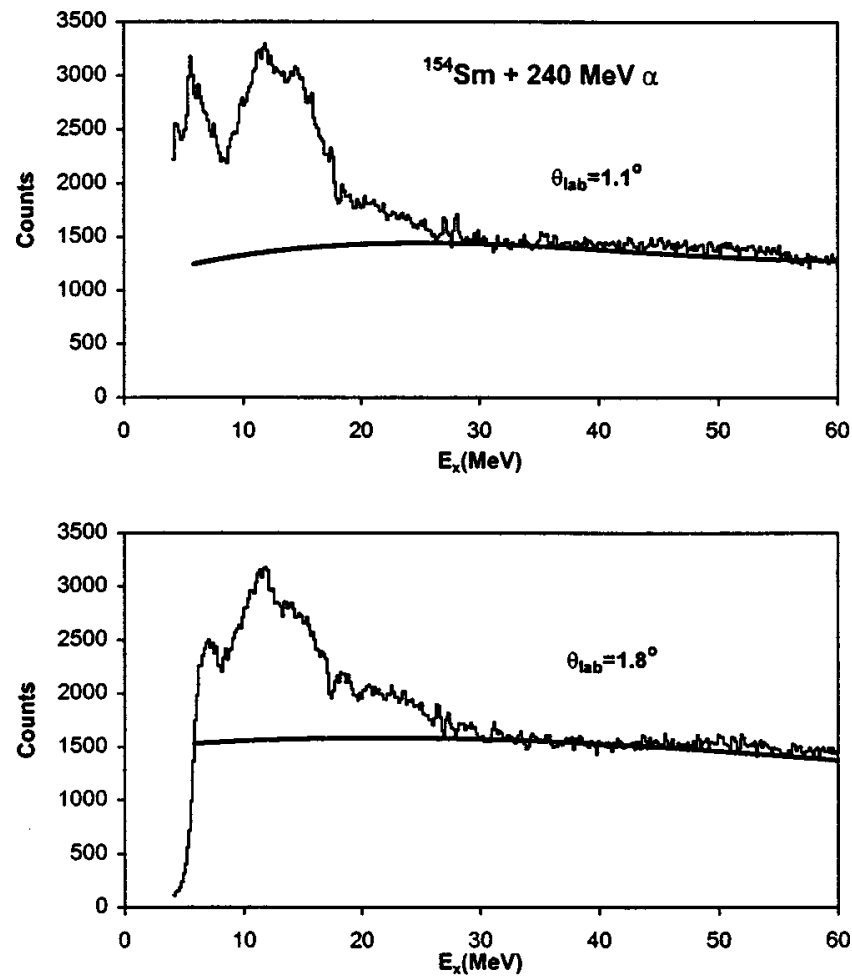

FIG. 1. Inelastic $\alpha$ spectra at two angles for ${ }^{154} \mathrm{Sm}$. The solid lines show the continuum chosen for the analysis.

$=20 \mathrm{MeV}$. The spectrum was divided into a peak and a continuum by extrapolating a line which at high excitation fits the continuum above the GR peak. These are indicated by the solid lines in the figure. This giant resonance (GR) peak is known to contain $L=0$ and $2 T=0$ strength and $L=1 T$ $=1$ strength [3] and likely contains $L=1$ and $L=3 T=0$ strength. Most past analyses have assumed that each component is present in a Gaussian-like peak and have done multipeak fits to separate the multipoles, depending on the differing behavior of each component with angle. The assumption that these resonances have a Gaussian shape has no theoretical basis and is contrary to most calculations of the strength distributions.

Thus the multipole components of the giant resonance peak were obtained by dividing the peak into multiple regions (bins) by excitation energy and then comparing the angular distributions obtained for each of these bins to distorted-wave Born approximation calculations to obtain the multipole components. A sample of the angular distributions obtained for the GR peak are shown in Figs. 2(a) and 2(b) while a sample angular distribution obtained for the assumed continuum is shown in Fig. 2(c). Similar analyses have been reported for ${ }^{24} \mathrm{Mg}$ [13] and ${ }^{28} \mathrm{Si}$ [12].

The transition densities and sum rules for various multipolarities are described thoroughly by Satchler [14] and the versions used in this work are given in Ref. [11]. Optical model parameters obtained for ${ }^{116} \mathrm{Sn}$ [15] were used in the deformed potential model calculations with the code PTOLEMY [16]. Input parameters for PTOLEMY were modified [17] to obtain a relativistic kinematically correct calculation. Radial moments were obtained by numerical integration of
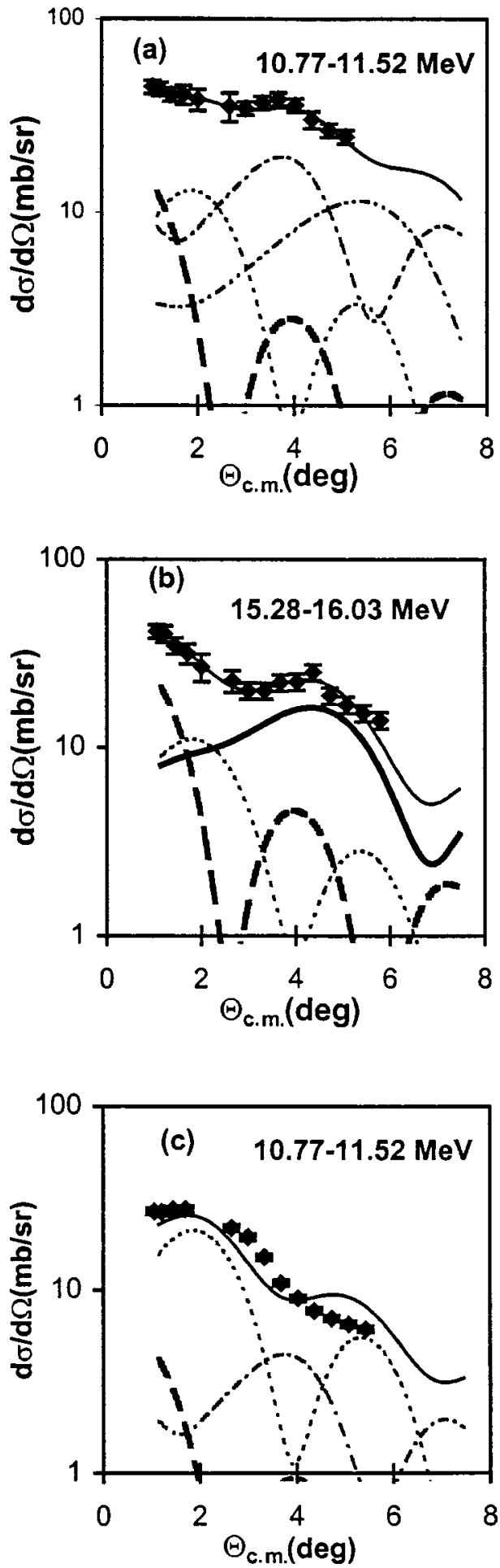

FIG. 2. Angular distributions of the differential cross section for inelastic $\alpha$ scattering for two excitation ranges of the giant resonance peak (a) and (b) and one range for the continuum (c) in ${ }^{154} \mathrm{Sm}$ plotted versus average center-of-mass angle. The solid lines show the sum of the distributions for the individual multipolarities. The dashed line shows the $L=0$ component, the dash-dotted line shows the $L=2$ component, the dotted line shows the $L=1 T=0$ component, the wide black line shows the $L=3$ component, and the dashdot-dot line shows the $L=4$ component for each of the regions. When not shown, errors are smaller than the data points. 

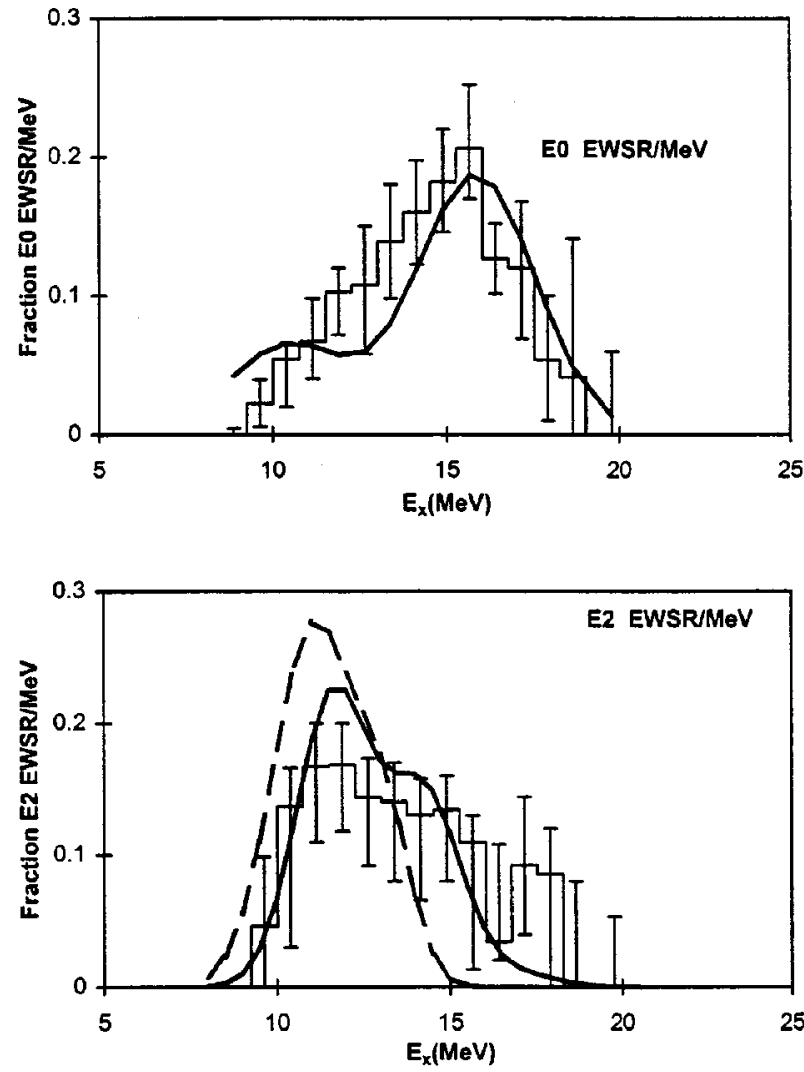

FIG. 3. The fractions of the $E 0$ and $E 2$ isoscalar EWSR in ${ }^{154} \mathrm{Sm}$ are shown by the histograms. The error bars represent the uncertainty due to the fitting of the angular distributions as described in the text. The thick lines are the predictions of Abgrall et al. [8], while the dashed line shows the prediction of Suzuki and Rowe [6].

the Fermi mass distribution with $c=6.107 \mathrm{fm}$ and $a$ $=0.523$.

Fits to the angular distributions obtained from the peak were carried out with a sum of isoscalar $0^{+}, 1^{-}, 2^{+}, 3^{-}$, and at higher excitation, $4^{+}$strengths. The isovector giant dipole resonance contribution is relatively small but was calculated from the known distribution [18] and held fixed in the fits. The strengths of the multipoles were varied to minimize $\chi^{2}$. The errors in strengths were estimated by changing the magnitude of the strength of one component until refitting by varying the other components resulted in a $\chi^{2}$ twice that of the best fit. The fits obtained along with the individual components of the fits are shown superimposed on the data in Fig. 2. The continuum angular distributions could not be fit with a sum of multipole strengths, suggesting that other processes dominate the continuum. The best fit obtained is shown in Fig. 2(c).

The $E 0$ and $E 2$ strength distributions obtained for the giant resonance peak are shown in Fig. 3. The errors obtained as described above are shown. Uncertainties due to the separation of the peak and continuum are not included. Both distributions are quite asymmetric and the $E 0$ distribution contains $\left(104_{-20}^{+15}\right) \%$ of the $E 0$ energy-weighted sum rule (EWSR), while the $E 2$ distribution contains $\left(103_{-20}^{+18}\right) \%$ of the E2 EWSR. The $L=1 T=0$ strength is distributed

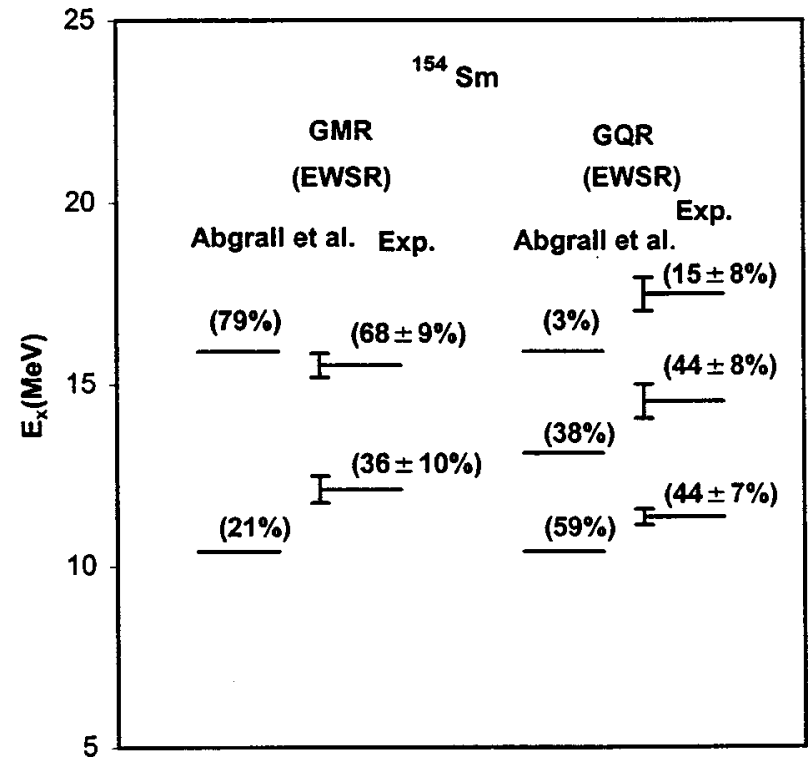

FIG. 4. Comparison of the predictions by Abgrall et al. [8] to the parameters for the fits to the distributions shown in Fig. 3.

roughly uniformly from $E_{x}=10-25 \mathrm{MeV}$ while the higher multipole strength (the data does always permit a reliable distinction between $L=3$ and 4 ) is distributed evenly between $E_{x}=8$ and $30 \mathrm{MeV}$. To provide a rough quantitative comparison with theory, the $E 0$ and $E 2$ distributions were fitted with the predicted number of Gaussian peaks (two for $E 0$ and three for E2), varying the position and strength of each independently, but constraining the widths of each component to be the same for $E 0$ and $E 2$ separately. The $E 0$ distribution was fitted with two Gaussians at $E_{x}=12.1$ $\pm 0.4 \mathrm{MeV}$ and $15.5 \pm 0.3 \mathrm{MeV}$ containing $(36 \pm 10) \%$ and (68 \pm 9$) \%$ of the EWSR, while the $E 2$ distribution was fitted with three Gaussians at $E_{x}=11.3 \pm 0.2 \mathrm{MeV}, \quad 14.5$ $\pm 0.5 \mathrm{MeV}$, and $17.5 \pm 0.5 \mathrm{MeV}$ containing (44 \pm 7$) \%$, (44 $\pm 8) \%$, and $(15 \pm 8) \%$, respectively, of the E2 EWSR. The earlier models for the GQR splitting (Kishimoto et al. [1], Suzuki and Rowe [6], Auerbach and Yeverechyahu [5], and Zawischa, Speth and Pal [7]) do not agree with the data, generally predicting a much smaller splitting than observed.

The strength distributions calculated by Adgrall et al. and by Suzuki and Rowe are shown superimposed on the data in Fig. 3. The calculation for the GQR by Suzuki and Rowe is much too narrow while that of Abgrall et al. agrees reasonably well with the data, though the experimental splitting appears a little larger than predicted and the highest ( $K$ $=2$ ) component is stronger than predicted. The GMR distribution calculated by Abgrall et al. is in reasonable agreement with the data though the experimental splitting is somewhat less than the calculation. The positions and strengths of the components extracted from the data are compared with those predicted by Adgrall et al. in Fig. 4 and are in fair agreement.

This work was supported in part by the U.S. Department of Energy under Grant No. DE-FG03-93ER40773 and by the Robert A. Welch Foundation. 
[1] T. Kishimoto, J. M. Moss, D. H. Youngblood, J. D. Bronson, C. M. Rozsa, D. R. Brown, and A. D. Bacher, Phys. Rev. Lett. 35, 552 (1975).

[2] H. Miura and Y. Torizuka, Phys. Rev. C 16, 1688 (1977).

[3] U. Garg, P. Bogucki, J. D. Bronson, Y.-W. Lui, C. M. Rozsa, and D. H. Youngblood, Phys. Rev. Lett. 45, 1670 (1980).

[4] H. P. Morsch, M. Rogge, P. Turek, C. Mayer-Boricke, and P. Decowski, Phys. Rev. C 25, 2939 (1982).

[5] N. Auerbach and Yeverechyahu, Phys. Lett. 62B, 143 (1976).

[6] Toskio Suzuki and D. J. Rowe, Nucl. Phys. A289, 461 (1977).

[7] D. Zawischa, J. Speth, and D. Pal, Nucl. Phys. A311, 445 (1978).

[8] Y. Abgrall, B. Morand, E. Caurier, and N. Grammaticos, Nucl. Phys. A346, 431 (1980).

[9] S. Jang, Nucl. Phys. A401, 303 (1983).

[10] R. De Leo, S. Brandenburg, A. G. Drentje, M. N. Harakeh, H. Janszen, and A. van der Woude, Nucl. Phys. A441, 591
(1985).

[11] D. H. Youngblood, Y.-W. Lui, and H. L. Clark, Phys. Rev. C 55, 2811 (1997).

[12] D. H. Youngblood, H. L. Clark, and Y.-W. Lui, Phys. Rev. C 57, 1134 (1998).

[13] D. H. Youngblood, Y.-W. Lui, and H. L. Clark, Phys. Rev. C 60, 014304 (1999).

[14] G. R. Satchler, Nucl. Phys. A472, 215 (1987).

[15] H. L. Clark, D. H. Youngblood, and Y.-W. Lui, Nucl. Phys. A589, 416 (1995).

[16] M. Rhoades-Brown, M. H. Macfarlane, and S. C. Pieper, Phys. Rev. C 21, 2417 (1980); M. H. Macfarlane and S. C. Pieper, Argonne National Laboratory Report No. ANL-76-11, Rev. 1, 1978 (unpublished).

[17] G. R. Satchler, Nucl. Phys. A540, 533 (1992).

[18] S. S. Dietrich and B. L. Berman, At. Data Nucl. Data Tables 38, 199 (1988). 